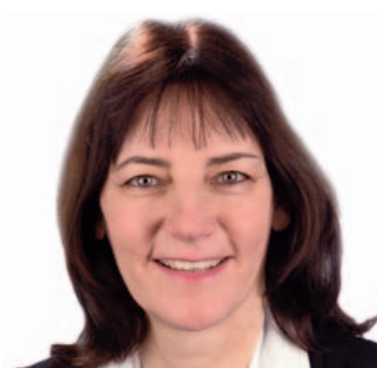

\title{
Pleura- und Lungenerkrankungen durch Asbest: Die Erkrankten von heute durch die Fehler von gestern
}

Alexandra M. Preisser

Universitätsklinikum Hamburg-Eppendorf, Zentralinstitut für Arbeitsmedizin und Maritime Medizin, Hamburg

Abstractübersetzung aus Mencoboni M, Filiberti RA, Taveggia P, Grosso F, Pasello G, Del Corso L, Muzio A, Polo V, Zucali P, Ceresoli GL, Soto Parra HJ, Auriati L, Simonassi C: Clinical features and treatment outcome of malignant pleural mesothelioma. Oncol Res Treat 2017:40:364-369.

Klinische Merkmale und Behandlungsergebnisse des malignen Pleuramesothelioms

\section{Schlüsselwörter}

Malignes Pleuramesotheliom · Chemotherapie · Chirurgie . Ansprechen · Überleben

\section{Zusammenfassung}

Hintergrund: Das maligne Pleuramesotheliom ist eine problematische Erkrankung, da die Prognose ungünstig und das Management schwierig ist. Wir evaluierten die Behandlung und den Verlauf von 378 Patienten mit Mesotheliom, die in sechs italienische Onkologiekliniken überwiesen wurden.

Methoden: Erhoben wurden demographische und klinische Daten. Als therapeutische Maßnahmen wurden Chemotherapie (Therapielinie, Pemetrexed-haltiges oder anderes Therapieschema), Chirurgie und Strahlentherapie in Betracht gezogen. Schließlich wurden Therapieansprechen und progressionfreies sowie Gesamtüberleben evaluiert.
Ergebnisse: 36 Patienten erhielten Best Supportive Care und 342 eine aktive Behandlung; davon unterzogen sich 86 einer Operation, bei 26 wurde eine trimodale Therapie angewendet. Mit der Erstlinienchemotherapie wurde bei 74,2\% der Patienten Krankheitskontrolle erreicht (75,7\% unter Behandlung mit Pemetrexed in Kombination mit anderen Wirkstoffen und 69\% unter Pemetrexed-Monotherapie). Bei Patienten mit wiederholter Pemetrexed-haltiger Therapie betrug die Krankheitskontrollrate 82,6\%. Die mediane Überlebensdauer betrug 11,6 Monate bei rein supportiver Therapie, 16,2 Monate bei reiner Chemotherapie, 32,4 Monate bei Chirurgie plus Chemotherapie und 47,2 Monate bei trimodaler Therapie. Eine günstigere Prognose wurde bei Patienten erreicht, die auf die Erstlinientherapie ansprachen und nachfolgend aktiv mit einer zweiten (24,8 vs. 11,8 Monate bei Nonrespondern; $p<0,001)$ und dritten Chemotherapielinie (28,9 vs. 17,8 Monate bei Nonrespondern; $p=0,05$ ) behandelt wurden.

Schlussfolgerung: Patienten mit Mesotheliom profitierten von einer alleinigen Chemotherapie nur, wenn sie nach Ansprechen auf die erste Therapielinie eine zweite erhielten.

(c) 2017 S. Karger GmbH, Freiburg

\section{KARGER}

(C) 2017 S. Karger GmbH, Freiburg 


\section{Transfer in die Praxis}

\section{Studienergebnisse}

Die Autoren untersuchten in ihrer Studie die Wirksamkeit und Bedeutung der verschiedenen Therapiemöglichkeiten des malignen Pleuramesothelioms: Erst-, Zweit und Drittlinienchemotherapie, Chirurgie, Strahlentherapie, auch in Kombinationen, oder Best Supportive Care. Der Artikel gibt einen systematischen Überblick zur Nachbeobachtung von 378 vorwiegend männlichen (74,3\%) Mesotheliomerkrankten aus sechs italienischen Kliniken.

Die Beobachtungen bestätigen frühere Ergebnisse: Die Überlebensrate ohne Therapie liegt unter einem Jahr und kann durch Chemotherapie durchschnittlich um vier Monate verlängert werden. Besonders bedeutsam ist die Wirkung der Erstlinientherapie: Zeigt sich auf die erste Chemotherapie ein Ansprechen (im Kollektiv wurde fast immer eine Pemetrexed-haltige Therapie gewählt), so profitieren diese Patienten auch von einer Zweitlinien- (meist Vinorelbine) oder Drittlinientherapie mit Überlebenszeiten von im Durchschnitt über zwei Jahren. War im Rahmen der hier vorgestellten Studie kein Ansprechen auf die erste Chemotherapie erkennbar, so wurde das Überleben auch nicht durch eine zweite oder dritte Therapie gesteigert, sondern lag im untersuchten Kollektiv unter einem Jahr. Ein erstes Ansprechen auf eine Chemotherapie bedeutet also mehr als eine Verdopplung der Lebenserwartung von 9 Monaten auf über 20 Monate im Durchschnitt. Bei einem resektablen Tumor wirkte sich auch die initale Pleurektomie und Dekortikation günstig auf die Überlebenszeit aus; die erweiterte Pneumonektomie zeigte jedoch eine erhöhte Frühmortalität. Die längsten Überlebenszeiten konnten bei geringer Tumorausdehnung und gutem Allgemeinzustand durch die trimodale Therapie mit Pleurektomie/Dekortikation, postoperativer Strahlentherapie und Chemotherapie erreicht werden (47 Monate; Range 37,5-56,9 Monate).

Die Autoren konnten Prognosefaktoren für ein längeres Überleben über 3 Jahre identifizieren: Alter <70 Jahre, epitheloidzelliger Tumortyp und guter Allgemeinzustand. Eine eingeschränkte Prognose zeigten Patienten mit erhöhtem LDH-Wert ( $>500 \mathrm{U} / \mathrm{I})$, erhöhter Thrombozytenzahl $\left(>400 \times 10^{3} / \mu l\right)$ oder negativem Calretinin Staging, die beiden ersteren erwiesen sich als statistisch unabhängige Prognosefaktoren.

\section{Fazit für die Praxis}

\section{Aktuelle Zahlen zum Pleuramesotheliom}

Die von Mencoboni et al. vorgelegte Studie ist angesichts der trotz EU-weitem Asbestverbot seit 2005 - zunehmenden Fälle von Pleuramesotheliom-Erkrankungen als Signaltumor für eine frühere Asbestexposition von besonderer praktischer Relevanz [1]. Die erheblichen beruflichen Expositionen gegenüber Asbest in Deutsch- land in den 1960er bis 1980er Jahren zeigen aufgrund der Latenz der Asbest-bedingten Pleura- und Lungenerkrankungen erst heute hohe Erkrankungszahlen mit hohen Behandlungs- und Kompensationszahlungen und viel Leid bei den Betroffenen. Die Berufskrankheitenstatistik registriert in den letzten Jahren stetig steigende Fallzahlen des Pleuramesothelioms; in 2015 wurde das bisherige Maximum mit 1040 Neuerkrankungen der entsprechenden Berufskrankheit (BK) Nr. 4105 erreicht [2]. Auch wenn für 2015 wieder ein leichter Rückgang auf 951 Fälle zu verzeichnen war, zeigte sich 2016 ein erneuter Anstieg auf 1031 Fälle [2]. Es bleibt im Verlauf abzuwarten, ob die Spitze der Asbest-bedingten Erkrankungen bereits erreicht wurde. Auch die Kosten für die Asbest-bedingten Erkrankungen sind erheblich, sie machten in 2015 insgesamt ca. ein Drittel aller BK-Kosten der deutschen Unfallversicherungsträger aus (BK 4105: 266,4 Mio. EUR; BK 4104: 231,2 Mio. EUR; BK 4103: 80,6 Mio. EUR) [3].

\section{Therapieempfehlungen}

Manceboni und Kollegen zeigen die Komplexität der Behandlung des Pleuramesothelioms auf. Nach Meinung und Erfahrung der Autoren sollte eine initiale Chemotherapie immer angestrebt werden. Wenn auf die erste Substanz jedoch kein Tumorrückgang feststellbar ist, raten die Autoren von Zweit- und Drittlinientherapien ab. Hingegen kann für ausgewählte Patienten mit resektablem Tumor die chirurgische Entfernung eine Option darstellen; jedoch ist auch hier - unter Berücksichtigung früherer Studien - die Überlebenszeit eingeschränkt (7,1 bis 33 Monate). Durch die dargestellten multimodalen Therapiekonzepte kann ein deutlich längeres Überleben der Patienten mit malignem Pleuramesotheliom erreicht werden.

\section{Disclosure Statement}

Hiermit erkläre ich, dass keine Interessenskonflikte in Bezug auf den vorliegenden Wissenstransfer bestehen.

\section{Literatur}

1 Karabin-Kehl B, Harth V, Preisser AM: Epidemiologie und arbeitsmedizinische Aspekte des Pleuramesothelioms. Pneumologie 2013; 67:209-218.

2 Anerkannte Berufskrankheiten 2016. Deutsche Gesetzliche Unfallversicherung (DGUV) 2017. www.dguv.de $\rightarrow$ Zahlen und Fakten $\rightarrow$ BK-Geschehen $\rightarrow$ Anerkannte BKen. (Zugriff 05.09.2017).

3 BK-Monitoring Bericht 2017. Berufskrankheiten im Jahr 2015. Deutsche Gesetzliche Unfallversicherung (DGUV), Berlin 2017.

Kontaktadresse: PD Dr. Alexandra M. Preisser, Universitätsklinikum Hamburg-Eppendorf, Zentralinstitut für Arbeitsmedizin und Maritime Medizin, Seewartenstraße 10, 20459 Hamburg, Deutschland, a.preisser@uke.de 